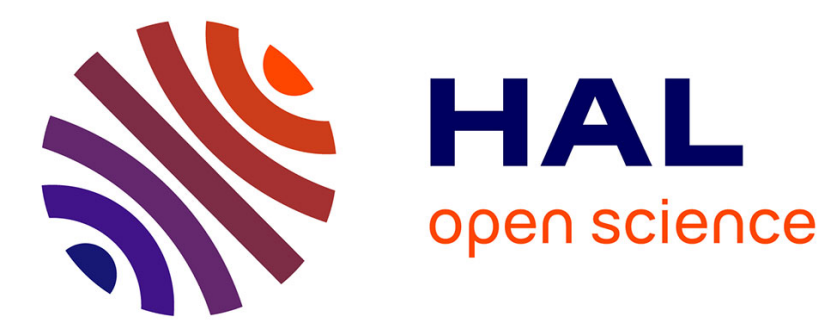

\title{
TRANSITOIRE D'ATTAQUE DES INSTRUMENTS À EMBOUCHURE DE FLÛTE
}

\author{
Benoît Fabre, A. Hirschberg, A. Wijnands, Van Steenbergen
}

\section{To cite this version:}

Benoît Fabre, A. Hirschberg, A. Wijnands, Van Steenbergen. TRANSITOIRE D'ATTAQUE DES INSTRUMENTS À EMBOUCHURE DE FLÛTE. Journal de Physique IV Proceedings, 1992, 02 (C1), pp.C1-67-C1-70. 10.1051/jp4:1992110 . jpa-00251053

\section{HAL Id: jpa-00251053 https://hal.science/jpa-00251053}

Submitted on 1 Jan 1992

HAL is a multi-disciplinary open access archive for the deposit and dissemination of scientific research documents, whether they are published or not. The documents may come from teaching and research institutions in France or abroad, or from public or private research centers.
L'archive ouverte pluridisciplinaire HAL, est destinée au dépôt et à la diffusion de documents scientifiques de niveau recherche, publiés ou non, émanant des établissements d'enseignement et de recherche français ou étrangers, des laboratoires publics ou privés. 


\title{
TRANSITOIRE D'ATTAQUE DES INSTRUMENTS À EMBOUCHURE DE FLUTTE
}

\author{
B. FABRE, A. HIRSCHBERG*, A.P.J. WIJNANDS* et A. VAN STEENBERGEN* \\ Laboratoire d'acoustique musicale, Université Paris 6, URA 868 du CNRS, 4 place Jussieu, \\ F-75005 Paris, France \\ ${ }^{*}$ Department of Physics, Eindhoven, University of technology W\&S 05, postbus 53, 5600 \\ $M B$ Eindhoven, The Netherlands
}

\section{ABSTRACT}

We propose a simple model to describe the transient of our experimental recorder. The acoustic energy due to the formation of the jet, combined with an estimation of the steady-state oscillation amplitude and of the linear gain of the system, predicts a rise time for the oscillation of the jet varying Erom 0.5 to $3 \mathrm{~ms}$ after the jet has reached the labium.It is only during this time that an edge-tone can coexist on the jet: there is no possible superposition while the jet oscillation is saturated. Once the jet oscillation is saturated, the model predicts a rise time for the oscillation in the pipe of the order of $0.1 s$. Our model shows a threshold (minimum driving pressure for oscillation) three times smaller than the experimental one. It also predicts the well known phenomena of higher mode transient typical for organ pipe.

\section{Introduction}

En utilisant des modèles simples, nous prévoyons le comportement en régime transitoire de notre flute expérimentale. Quatres phases temporelles sont identifiables dans nos mesures [1] grâce à la visualisation et aux relevés de pression dans le pied du tuyau et au niveau du biseau (Figure 1).

Dans la première phase (quelques millisecondes) le jet '́merge du canal et progresse vers le biseau. Durant cette phase, avant d'atteindreme biseau, le jet se comporte comme une source de volume injecté dans le système imóépendamment de la présence du biseau. Cette injection apporte une quantité initiale d'énergie acoustique à l'instrument.

Dans la seconde phase du transitoire le jet atteint le biseau. Deux phénomènes apparaissent alors: d'une part des oscillations de fréquences élevées qui disparaissent dans la phase suivante (dans notre montage expérimental, ces oscillations sont dominés par une composante à la première fréquence de résonance transversale du tuyau $(6 \mathrm{kHz})$ ) et d'autre part la croissance d'une oscillation à une fréquence proche d'une fréquence de résonance longitudinale du tuyau.

La troisième phase est celle de la croissance de l'amplitude de la pression dans le tuyau: le jet est alors en saturation et se comporte comme une source d'amplitude constante. La durée de cette phase est donc proportionnelle au coefficient de surtension du tuyau pour le mode considéré.

La dernière phase est celle de la saturation du système: les mécanismes de perte limitent l'amplitude de l'oscillation et provoquent la stabilisation sur un mode longitudinal du tuyau souvent different de celui observé dans les phases précédentes.

2 Modèle

A partir de la mesure de la différence de pression entre le pied et le tuyau, nous calculons la vitesse de l'écoulement en sortie du canal par intégration de 
l'équation de Bernoulli non-stationnaire qui prend en compte les effets d'inertie dans le canal. Le spectre obtenu par transformation de Fourier du signal de vitesse détermine, avec la réponse du tuyau pour une injection de débit, les conditions initiales de pression dans le tuyau.

I'énergie apportée au système durant cette première phase temporelle est concentrée aux basses fréquences et ne permet donc pas de prévoir un comportement à hautes fréquences comme le mode transversal que l'on observe dans la seconde phase. En effet, ces oscillations semblent liées à des phénomènes hydrodynamiques complexes de turbulence qui apparaissent après que le jet ait atteint le biseau.

Nous négligerons en première approximation l'influence de ces "hautes fréquences" sur la suite du développement et nous supposerons un comportement linéaire du jet. A noter que cette hypothèse de linéarité permet d'imaginer, en plus du son de tuyau étudié ici, la coexistence d'autres régimes d'oscillation sur le jet, comme un son de biseau.

La croissance de l'oscillation du jet à une fréquence proche d'un mode propre longitudinal du tuyau peut être décrite en utilisant un modèle semi-empirique. Nous supposons le jet formé et ayant acquit sa vitesse stationnaire; il est alors soumis aux perturbations dues à l'énergie acoustique accumulée aux fréquences propres du tuyau pendant la première phase. Nous utilisons la formulation retenue lors de nos expériences en régime stationnaire: les résultats obtenus par Mattingly \& Criminale [2] (coefficient d'amplification $A$ et vitesse de propagation $u$ sur le jet) sont combinés avec la formule empirique de Fletcher [3] pour décrire la position du jet au biseau $\eta$ en fonction de la vitesse acoustique $v_{a}$ dans la bouche de l'instrument:

$$
\eta(t)=\frac{v_{a} e^{j \omega t}}{j \omega} \cosh \left(\frac{a}{b}\right) e^{-j \omega \frac{d}{u}}
$$

si d est la distance lumière-biseau et b le paramètre d'épaisseur du jet ( $b=0,4 h$ pour un profil de vitesse de Poiseuille si h est l'épaisseur du canal).

Le jet oscille au niveau du biseau, crént deux sources de volume complémentaires en opposition de phase de part et d'autre du biseau. I'ensemble de ces deux sources possède un comportement dipolaire dont nous considérerons la limite atteinte lorsque la source externe est située à un point d'impédance acoustique nulle. Nous obtenons de la sorte une expression analogue à la formulation proposée par Fletcher [3] pour la réponse du tuyau: la vitesse acoustique va dans la bouche est donnée en fonction du débit $Q_{j}$ injecté par le jet dans le tuyau par:

$$
v_{a}=\frac{Q_{j}}{S_{m}} \frac{Z_{m}}{z_{p}+z_{m}}
$$

où $z_{m}$ et $z_{p}$ sont les impédances respectives de la bouche et du tuyau vues depuis le point d'injection et $S_{m}$ est la surface de la bouche.

Durant cette phase, nous supposons que le déplacement latéral du jet au niveau du biseau est petit devant son épaisseur $h$, aussi nous considérons une dépendance linéaire de la source $Q_{j}$ en fonction de la position du jet $\eta$. Lë système est alors étudié en utilisant le formalisme des systèmes bouclés linéaires. L'instabilité d'un tel système peut être prévue d'après I'étude du gain $G$ en boucle ouverte du système:

$$
G(j \omega)=\frac{v_{j} D}{j \omega S_{m}} \cosh \left(\frac{A}{b}\right) e^{-j \omega \frac{d}{u}} \frac{z_{m}}{z_{p}+z_{m}}
$$

où $D$ est la largeur du jet

Les fréquences instables (pour lesquelles une petite perturbation sera amplifiée) sont telles que:

$$
|G(j \omega)| \geq 1 \quad(4 a)
$$

$\Phi(G(j \omega))=2 n \pi$ avec $n$ entier $(4 b)$

\section{Résultats}

En utilisant les valeurs des paramètres qui correspondent à notre flûte expérimentale, nous calculons, pour chaque valeur de la vitesse du jet, les fréquences instables. Les fréquences instables obtenues sont présentées figure 2 en fonction de la vitesse du jet. Dans le cas ou plusieurs fréquences instables apparaissent, nous ne retenons que celle pour laquelle le gain $G$ est le plus fort. 
En effet, plus le gain $G$ est important, plus la croissance du mode instable sera rapide. Le transitoire donné par notre modele est donc d'autant plus rapide que la fréquence $d^{\prime}$ instabilité est proche $d^{\prime}$ une fréquence de résonance passive du tuyau (maximum du gain $G$ ).

La figure 2 montre que la fréquence instable augmente très légèrement avec la vitesse du jet puis saute brutalement au mode supérieur. Les points obtenus à basse vitesse correspondent aux situations pour lesquelles le jet est long de plus d'une longueur d'onde hydrodynamique. Par ailleurs, notre modèle permet aussi de prévoir un seuil de fonctionnement pour les basses vitesses de jet. Le seuil obtenu figure 2 est de $4 \mathrm{~m} / \mathrm{s}$ pour le premier régime soit une pression d'alimentation d'environ 10 Pa d'après l'equation de Bernoulli, alors que le seuil expérimental est de $30 \mathrm{~Pa}$. A partir de $8 \mathrm{~m} / \mathrm{s}(40 \mathrm{~Pa})$, le second mode est le plus instable en régime linéaire. Ceci explique en partie le démarrage sur le second partiel observé dans nos expériences. Il faut noter que la position asymétrique du biseau par rapport à la lumière dans notre montage provoque une saturation dissymétrique du jet, favorisant aussi le démarrage sur un partiel supérieur.

A partir de la fréquence d'instabilité $f$, du gain $G$ et des amplitudes relatives des pressions po due a l'impact initial (phase 1) et pmax du régime stationnaire, nous pouvons majorer le temps de montée $t$ du modéle:

$$
t<\frac{1}{f} \frac{\log \left(\frac{p_{\max }}{p_{0}}\right)}{\log (G)}
$$

ou pmax est calculé en considérant la réponse du tuyau à une source de volume, fonction "carré" en saturation totale:

$$
Q_{j}= \pm \frac{V_{j} D h}{2}
$$

Les temps de montée calculés varient de 3ms pour les basses vitesses de jet $(5 \mathrm{~m} / \mathrm{s})$ à $0,5 \mathrm{~ms}$ pour les vitesses élevées (démarrage sur le troisième mode). Il faut cependant noter que les valeurs de $p_{\max }$ calculées sont 2 a 10 fois supérieures aux valeurs mesurées suivant les conditions. Ceci indique que la seule prise en compte des pertes par rayonnement et des pertes visco-thermiques est insuffisante. Les phénomènes de tourbillons observés dans nos visualisations ont été ignorés; ils sont susceptibles d'introduire des pertes importantes liées à l'amplitude de l'oscillation (mécanisme limiteur). De plus, l'approximation de Fletcher (source externe négligée) surestime l'amplitude de la source.

La durée de la dernière phase est évaluée par:

$t=T_{n} Q_{n}$

ou $T_{n}$ et $Q_{n}$ sont respectivement la période et le coefficient de surtension associés au mode $n$ sélectionné lors de la phase précédente (eq..4 \& 4b). Pour notre géométrie expérimentale, le calcul donne des temps de l'ordre de... $1 s$ pour les trois premiers partiels du tuyau, ce qui correspond aux valeurs expérimentales. On remarquera que le modele devrait surestimer ce temps puisqu'il sous-estime les pertes.

\section{Conclusions}

Nous retrouvons le phénomène connu de l'accrochage d'un régime supérieur du tuyau au moment du transitoire dans les tuyaux d'orgue: octaviation ou quintoiement. Le seuil de fonctionnement que nous prévoyons est trois fois plus faible que celui que nous mesurons à l'inverse du modèle proposé par Fletcher qui prévoyait un seuil trois fois trop haut.. Si un son de biseau apparait pendant la seconde phase, notre modèle prévoit qu'il ne dure que peu de temps.

Le découpage en phases temporelles doit être nuancé: le mécanisme d'injection considéré dans la première phase se prolonge puisque la vitesse du jet est affectée par l'oscillation dans le tuyau. Il faudra vérifier si la théorie semi-empirique du jet établie en régime stationnaire reste valable lors du transitoire. Les mécanismes de pertes considérés sont insuffisants et doivent être complétés. 
5 Bibliographie

[1] A. Hirschberg, A.P.J. Wijnands, A. van Steenbergen, J.J. de Vries, B. Fabre, J. Gilbert "Jet drive \& edge tone in flue organ pipes" Actes du "colloque modeles physiques", ACROE, INPG, Grenoble, France (1990)

[2] G.E. Mattingly \& W.O. Criminale, jr, "Disturbance characteristics in a plane jet" The physics of fluids 14, 2258-2264 (1971)

[3] N.H. Fletcher, "Sound production by organ flue pipes" J.Acoust.Soc.Am. 60, 926936 (1976)

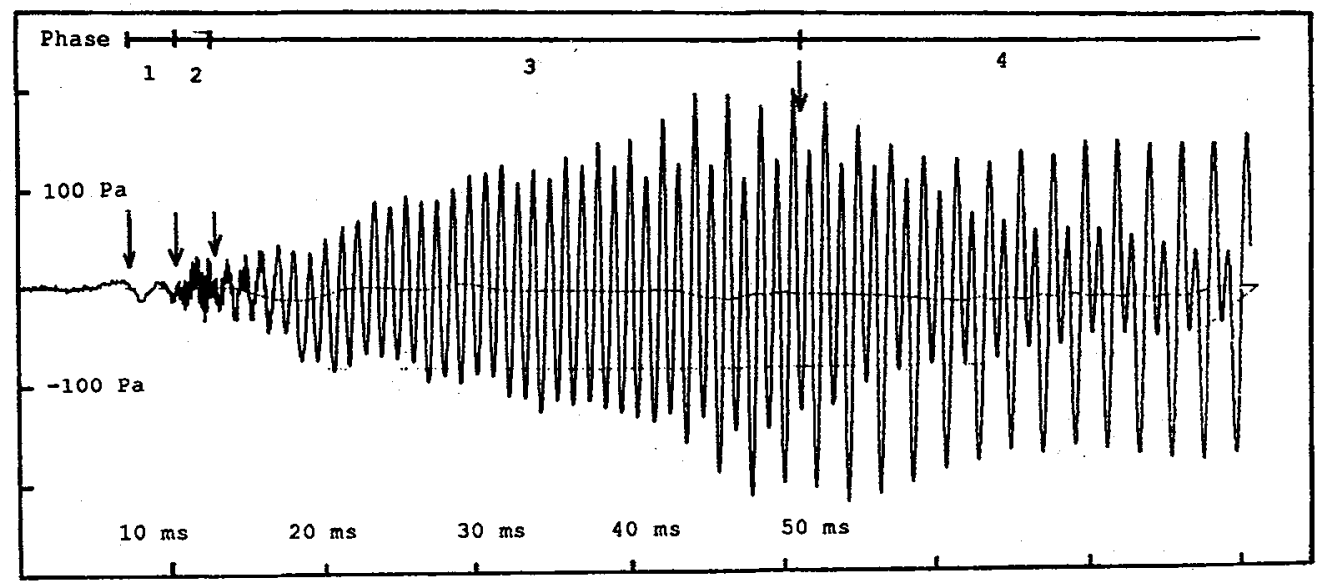

Figure 1 Phases temporelles du transitoire: signal de pression dans le tuyau face au biseau.

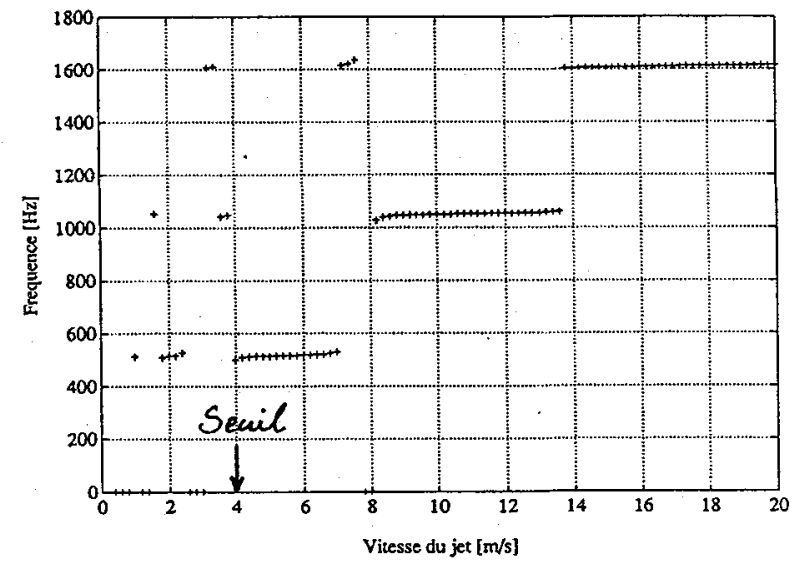

Figure 2 Analyse linéaire: prévision de la fréquence instable (Hz) en fonction de la vitesse du jet $(\mathrm{m} / \mathrm{s})$ 